\title{
Tetra ostial coronary anatomy- A rare pattern in transposition of great arteries.
}

\author{
Joanna Roy ${ }^{1}$, Sreeja Pavithran², and Roy Varghese ${ }^{2}$ \\ ${ }^{1}$ Topiwala National Medical College \\ ${ }^{2}$ Madras Medical Mission Institute of Cardio Vascular Diseases
}

May 28, 2021

\begin{abstract}
Meticulous transfer of coronary arteries is of crucial importance in transposition of great arteries and determines the success of the switch procedure. This report describes a coronary anatomy consisting of four separate ostia from the two facing sinuses in a six-month-old infant presenting with d-transposition of great arteries and ventricular septal defect. Being a rare coronary arterial pattern not described in previous coding systems, the surgeon would do well to be aware of this possibility while performing the switch procedure.
\end{abstract}

\section{TITLE PAGE}

TITLE: Tetra ostial coronary anatomy - A rare pattern in transposition of great arteries.

Running head: Rare coronary arterial anomaly in TGA.

Authors: Joanna Mary Roy, $\mathrm{MBBS}^{1}$, Sreeja Pavithran, $\mathrm{MD}^{2}$, Roy Varghese, $\mathrm{MCh}^{3}$

1. Medical student (MBBS), Topiwala National Medical College and BYL Nair Hospital, Mumbai, India

2. Senior consultant, Pediatric cardiology, Madras Medical Mission, Chennai, India

3. Senior consultant, Pediatric cardiac surgery, Madras Medical Mission, Chennai, India

Word count: 1270 .

Classifications: cardiac anatomy/pathologic anatomy; pediatric; congenital heart disease, CHD; CHD, great vessel anomalies; transposition, arterial switch.

\section{Address for correspondence:}

Roy Varghese

Senior consultant, Pediatric cardiac surgery,

Institute of Cardiovascular Diseases

Madras Medical Mission,

J.J Nagar, Chennai, India.

Postal code: 600037 .

Email: jonroy65@gmail.com

Sources of funding: None. 
Conflicts of interest: None.

IRB approval/ waiver: N/A

Informed consent: N/A

Clinical trial registration: $\mathrm{N} / \mathrm{A}$.

Data availability statement: Data will be made available upon request from the authors.

\section{ABSTRACT}

\section{Background}

Meticulous transfer of coronary arteries is of crucial importance in transposition of great arteries and determines the success of the switch procedure. This report describes a coronary anatomy consisting of four separate ostia from the two facing sinuses in a six-month-old infant presenting with d-transposition of great arteries and ventricular septal defect.

\section{Conclusion}

Being a rare coronary arterial pattern not described in previous coding systems, the surgeon would do well to be aware of this possibility while performing the switch procedure.

\section{INTRODUCTION}

Coronary arterial anatomy is of crucial significance during the switch procedure and post-operative recovery in transposition of great arteries (TGA). The anatomical variability of coronary arteries has been described using the Leiden Convention. This report illustrates a hitherto undescribed pattern of coronary artery anatomy that was encountered during the procedure.

\section{CASE REPORT}

A 6- month-old male child weighing $3.5 \mathrm{~kg}$ presented with failure to thrive and oxygen saturations of $75 \%$ on room air. Echocardiography confirmed d-transposition of great arteries with a subpulmonic ventricular septal defect (VSD). Single origin of coronary arteries with separate ostia from sinus 1 (Leiden Convention: 1R,LCx) was suspected and the child was scheduled for arterial switch operation and closure of VSD.

Standard median sternotomy was followed by harvest of a patch of pericardium that was promptly treated with $0.0625 \%$ glutaraldehyde solution for five minutes. Following heparinization, cardiopulmonary bypass was instituted with distal ascending aortic and bicaval venous cannulation. The temperature of the perfusate was cooled to 280C. Cold cardioplegic (Del Nido) arrest was achieved and the VSD was closed through the trans right atrial route using the previously harvested patch of pericardium and a continuous suture of 60 ' polypropylene. Following this, the aorta and main pulmonary arteries were transected at appropriate levels above the respective sinotubular junctions.

Inspection of the aortic sinuses confirmed the coronary anatomy pattern to be different from that previously described: Sinus 1 gave origin to a right coronary artery (RCA) supplying the anterior wall of the right ventricle. In addition, a separate ostium from the same sinus gave origin to the left coronary artery that divided after a short course into anterior descending and circumflex coronary arteries (Figure 1). Sinus 2 was also found to contain two coronary arteries arising through separate ostia; an RCA running in the right atrioventricular groove and an additional circumflex coronary artery coursing posterior to the pulmonary artery (posterior looping) to gain the posterior and lateral walls of the left ventricle (Figure 2).

The coronary anatomy pattern can therefore be described $1 \mathrm{R}, \mathrm{LCx}-2 \mathrm{R}, \mathrm{Cx}$ as per the Leiden Convention (Figure 3).

Two coronary buttons were excised separately as in the standard switch procedure including the respective ostia in either button. Following adequate mobilization (particularly for the RCA from sinus 1), the left sided button (sinus 1) was transferred to the neo aortic root. The coronary branches on the right sided button 
(sinus 2) required wider mobilization. Due to the posterior looping of the corresponding circumflex artery from sinus 2, this button was implanted higher up (two thirds of the button extending into the ascending aorta). There was significant size discrepancy between the neo aortic root and the ascending aorta; an incision into the ascending aorta to accommodate the right sided button helped to equalize this discrepancy.

The right ventricular outflow tract was reconstructed as in the standard switch procedure using the treated pericardium as a "pantaloon patch". The child was rewarmed and weaned off bypass uneventfully. He was weaned off the ventilator after 48 hours.

\section{COMMENT}

Successful transfer of the coronary arteries is the single most important step in the performance of the switch procedure for transposition of great arteries. While its effects are evident immediately at the termination of the procedure in almost all instances, subtle deficiencies become evident on follow up and manifest as poor ventricular function ${ }^{1}$.

The Leiden Convention identified the prevalent coronary artery patterns encountered and remain the most widely followed coding system to date ${ }^{2}$. Moll et al similarly described the coronary artery patterns in a large cohort of 700 patients and identified 16 types with their relative frequencies ${ }^{3}$. While no pattern of coronary anatomy per se is a contraindication for the switch procedure, these classifications and coding systems serve to prepare the surgeon on the technical modifications to be adopted while performing the operation.

Looping of coronary arteries is associated with poor outcome when they originate from a single ostium, while no significant risk has been reported in cases where the arteries take origin from separate sinuses ${ }^{4}$. Two ostia arising from a single sinus have been described by Moll et al and they identified it as one of the risk factors associated with poor outcome in the immediate post-operative period ${ }^{3}$. This report describes a patient with double ostia from both sinuses hitherto undescribed in the coding systems. While origin of the circumflex artery from the RCA is not associated with incremental risk, the patient in this report would be at higher than usual risk of a poor outcome on account of his double ostia from the two sinuses ${ }^{3}$. This situation was managed by adequate mobilization of the individual coronary arteries combined with higher seating of the right sided button on to the ascending aorta respecting the posterior looping of the circumflex coronary artery as described by Lacour-Gayet ${ }^{5}$.

Identification of the coronary artery pattern in the individual case plays a key role in the successful performance of the switch procedure. Awareness of the pattern described in this report prepares the surgeon for the possibility of dealing with four ostia while performing the coronary transfer.

\section{Author contributions:}

JMR: drafting the article, concept/design; SP: data collection, drafting the article; RV: critical revision of the article, concept/design, data acquisition.

\section{REFERENCES}

[1] Martin MM, Snider AR, Bove EL, et al. Two-dimensional and Doppler echocardiographic evaluation after arterial switch repair in infancy for complete transposition of the great arteries. Am. J. Cardiol.1989;63(5),332-336.

[2] Gittenberger-de Groot AC, Koenraadt WMC, Bartelings MM, et al. Coding of coronary arterial origin and branching in congenital heart disease: The modified Leiden Convention. J Thorac Cardiovasc Surg. 2018;156(6):2260-2269.

[3] Moll M, Michalak KW, Sobczak-Budlewska K, et al. Coronary artery anomalies in patients with transposition of the great arteries and their impact on postoperative outcomes. Ann Thorac Surg . 2017;104(5):16201628.

[4] Pasquali, S. K. Coronary artery pattern and outcome of arterial switch operation for transposition of the great arteries: A meta-analysis. Circulation. 2002;106(20), 2575-2580. 
[5] Lacour-Gayet F, Anderson RH. A uniform surgical technique for transfer of both simple and complex patterns of the coronary arteries during the arterial switch procedure. Cardiol Young.2005;15(Suppl.1):93101.

\section{FIGURE LEGENDS}

Figure 1: RCA and LCA from separate ostia in sinus 1.

Abbreviations: LCA: Left coronary artery, RCA: Right coronary artery.

Figure 2: RCA and circumflex artery taking origin from separate ostia in sinus 2.

Abbreviations: RCA: Right coronary artery.

Figure 3: Schematic diagram showing the coronary artery pattern as per the Leiden convention: (1R,LCx$2 \mathrm{R}, \mathrm{Cx})$.
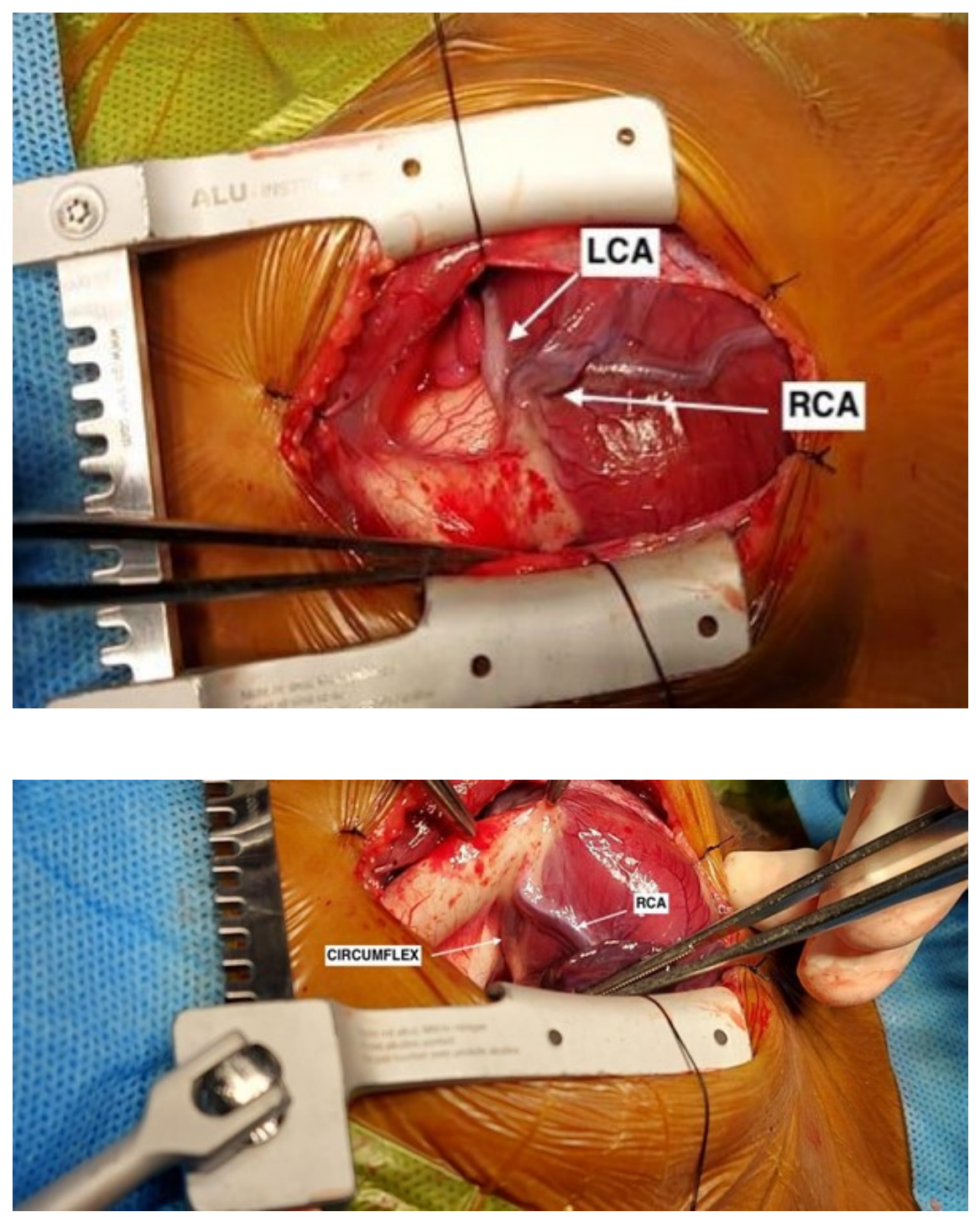


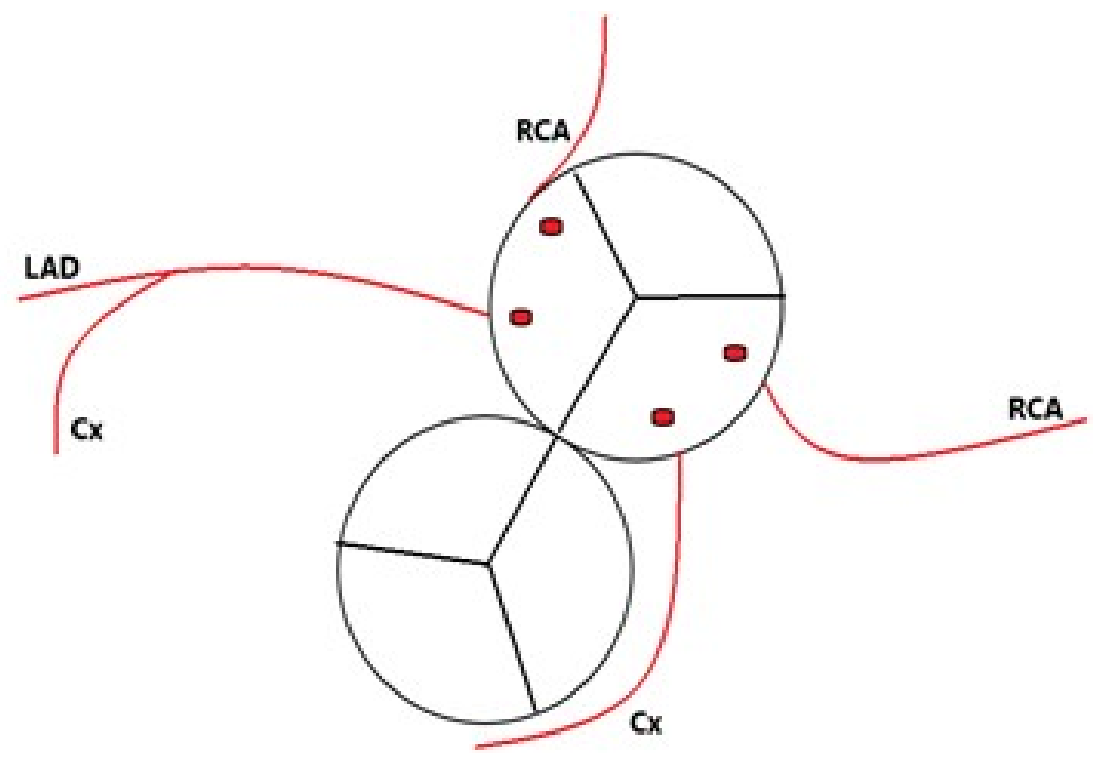

